\title{
Pro-tumorigenic roles of TGF- $\beta$ signaling during the early stages of liver tumorigenesis through upregulation of Snail
}

\author{
Hyuk Moon ${ }^{1}$, Kwang-Hyub Han $^{2}$ E Simon Weonsang Ro ${ }^{1, *}$ \\ ${ }^{1}$ Institute of Gastroenterology, ${ }^{2}$ Department of Internal Medicine, Yonsei University College of Medicine, Seoul 03722, Korea
}

\begin{abstract}
Many studies have focused on the tumor suppressive role of TGF- $\beta$ signaling during the early stages of tumorigenesis by activating the target genes involved in cytostasis and apoptosis. We investigated the effects of TGF- $\beta$ inhibition on early tumorigenesis in the liver, by employing diverse inhibitory methods. Strikingly, TGF- $\beta$ inhibition consistently suppressed hepatic tumorigenesis that was induced either by activated RAS plus p53 downregulation or by the co-activation of RAS and TAZ signaling; this demonstrates the requirements for canonical TGF- $\beta$ signaling in tumorigenesis. Moreover, we found that Snail is the target gene of the TGF- $\beta$ signaling pathway that promotes hepatic carcinogenesis. The knockdown of Snail suppressed the early tumorigenesis in the liver, as did the TGF- $\beta$ inhibition, while the ectopic expression of Snail restored tumorigenesis that was suppressed by the TGF- $\beta$ inhibition. Our findings establish the oncogenic TGF- $\beta$-SmadSnail signaling axis during the early tumorigenesis in the liver. [BMB Reports: Perspective 2017; 50(12): 599-600]
\end{abstract}

\section{TGF- $\beta$ TUMOR PROMOTION IN THE LIVER}

Although studies have shown that TGF- $\beta$ can function as tumor suppressors in a variety of tissues, including the liver, other lines of evidence suggest that TGF- $\beta$ signaling promotes spontaneous tumorigenesis in the liver. Fibrotic and cirrhotic livers are highly susceptible to the development of hepatocellular carcinomas (HCC), which are characterized by the

*Corresponding author. E-mail: simonr@yuhs.ac

https://doi.org/10.5483/BMBRep.2017.50.12.201

Received 26 September 2017

Keywords: Cancer model, Hepatocellular carcinoma, Snail, Transforming growth factor $\beta$ (TGF- $\beta$ ), Tumor promotion

Abbreviations: EMT, epithelial-to-mesenchymal transition; HCC, hepatocellular carcinoma; CCA, cholangiocarcinoma

Perspective to: Hyuk Moon et al. (2017) “Transforming Growth Factor Beta Promotes Liver Tumorigenesis in Mice via Upregulation of Snail." Gastroenterology 153(5):1378-1391. upregulation of TGF- $\beta$ signaling. Animal models show an increased spontaneous carcinogenesis in fibrotic livers which were induced by various fibrogenic factors, including TGF- $\beta 1$. It is largely unknown how the fibrotic/cirrhotic environment facilitates spontaneous liver tumorigenesis.

We have previously shown that activation of the Sonic Hedgehog $(\mathrm{SHH})$ signaling in the liver induced hepatic fibrosis and promoted HCC tumorigenesis (Chung et al., (2016) J Hepatol 64, 618-627). Notably, we found significant upregulation of TGF- $\beta$ signaling and epithelial-to-mesenchymal transition (EMT) phenotypes in fibrotic livers induced by $\mathrm{SHH}$ activation, prior to the development of HCC. A recent study by Hernanda et al showed that SMAD4 expression and its nuclear localization are significantly elevated in HCC tissues, as compared with adjacent liver tissues (Hernanda et al., (2015) Oncogene 34, 5055-5068). In a paper recently published in Gastroenterology, we demonstrated that TGF- $\beta$ signaling can promote primary carcinogenesis in the liver via the upregulation of an EMT inducer, specifically, the Snail. We found that the Snail promotes cellular proliferation, as well as plays a critical role in the initiation of primary tumorigenesis in the liver.

\section{THE ROLE OF THE SNAIL IN THE PRIMARY TUMORIGENESIS IN THE LIVER}

Our work shows that the Snail can initiate primary tumorigenesis of HCC and cholangiocarcinoma (CCA), in collaboration with an oncogene such as an activated RAS or TAZ. Furthermore, the downregulation of the Snail efficiently suppressed oncogene-induced primary tumorigenesis. Although the exact molecular mechanism by which the Snail promotes primary tumorigenesis in the liver remains to be determined, our data show that the Snail expression induced alterations in cell adhesion, tight junctions and the extracellular matrix, as well as the downregulation of E-cadherin. Disruption of tight junction proteins and E-cadherin can induce epithelial proliferation and trigger oncogenic signaling pathways. Notably, the genetic deletion of E-cadherin significantly contributed to liver tumorigenesis induced by activated RAS or a chemical carcinogen. Further studies are needed to elucidate the exact mechanism by which the Snail activates pro-tumorigenic signaling pathways in the liver. 


\section{CANCER INITIATION BY EMT INDUCERS}

Recent studies have shed new light on the role of EMT inducers in cancer initiation. EMT inducers provide cancer cells with invasive and metastatic capabilities, thus allowing for the initiation of cancer at new locations. We refer to metastatic cancer initiation as the "initiation of secondary tumorigenesis (IST)" to distinguish it from the initiation of primary tumorigenesis. The IST capability of cancer cells is often evaluated using xenograft models, in which tumor cells which overexpress EMT inducers demonstrate an increased potential for cancer initiation following transplantation.

Compared with IST, the role of EMT inducers in the initiation of primary tumorigenesis is less well known. Recently, however, several groups reported that Twist1 can promote spontaneous tumorigenesis in the lungs, skin, and mammary tissues. Twist1 has been shown to abrogate cellular senescence and apoptosis by repressing the $\mathrm{p} 16^{\mathrm{INK} 4 \mathrm{~A}}$ and p53 pathways, respectively. Supporting these inhibitory effects of Twist1 on senescence and apoptosis, the co-expression of Twist1 and activated RAS efficiently initiated the primary tumorigenesis in vivo. Additionally, the suppression of Twist1 expression reactivated the senescence and apoptotic programs, which suppressed initiation of the primary tumorigenesis in the skin and lung epithelial tissues. In addition to the well-known roles that these entities play in metastatic cancer initiation, these new-found roles of EMT inducers in the initiation of primary tumorigenesis signify the diverse oncogenic effects of EMT inducers.

\section{ACKNOWLEDGEMENTS}

This work was supported by the National Research Foundation of Korea (NRF) grant 2016R1A2B4013891 (awarded to S.W.R.), which was funded by the Korea government (MSIP). 